\title{
Study on the Knitted Garment Pattern Design Method and Its Application
}

\author{
Zhoumin Yang \\ Xi'an Peihua University, Xi’an, Shaanxi, 710125
}

Keywords: knitwear; pattern design; application

\begin{abstract}
With the continuous development of China's national economy, people's material consumption levels have been continuously rising, and the clothing industry has also developed, which poses higher requirements for apparel design work. Pattern design is an important basic link for apparel design. The pattern design can lay a good foundation for the garment design work because of its warm and comfortable fabric characteristics, and knitted fabrics are favored by consumers, which are also widely used in the design and production of garments. However, because of the special fabrics of knitted fabrics certain requirements have also been proposed for the design and production of garments. This paper deeply analyzes the design method of knitted garment pattern and its specific application to improve the design level and production level of knitted garments.
\end{abstract}

\section{Introduction}

Knitwear refers to garments made mainly of knitted fabrics. The fabrics have excellent characteristics such as elasticity, high extensibility, skin-friendly softness, and good breathability. Many designers like to incorporate knitted fabrics into their own apparel designs. This makes knitwear gradually become a new fashion form. Knitted fabrics are used in apparel design and have excellent characteristics such as retractability, stretchability and hemming, which can directly affect the design specifications and finished product styling of garments. It also makes it a major factor affecting pattern design. The pattern design method of the pattern is similar to the pattern design method of the woven garment. The main design methods include the prototype method, the three-dimensional cutting method, the specification algorithm, and the basic sample method. The specific design links and design methods are relatively complicated and need to be designed. Some people have a good understanding of the characteristics of knitted fabrics in order to design good patterns and clothing styles.

\section{Background of Knitted Fabric Design}

With the continuous development of social productivity, from the initial shortage of social goods to the relative surplus of affluence and finally to the present, consumers have a rational consumption concept from the initial lack of choice to the blind follow-up to the now established, knitwear market structure. Under the current situation of diversified demand and personalized trends triggered by the upgrading of consumption structure, how to accurately grasp the nature of the industry and the core needs of consumers, so as to effectively carry out the positioning of the crowd, to guide the consumer lifestyle, lead the fashion trend, Become the key to knitwear design [1].

\section{The Specific Impact of Knitted Fabric on Pattern Design}

Due to the special fabric properties of knitted fabrics, knitted garments have excellent breathability and hygroscopicity. The unique coil structure of knitted fabrics can absorb more air compared to other fabrics, so it is breathable, hygroscopic and warm. There are irreplaceable advantages of the same price apparel fabrics, people can intuitively feel its comfort when wearing, but also because of these advantages make it the first choice for underwear, sportswear, casual wear 
and other apparel fabrics. When the coil structure is subjected to external force, the fabric can be easily stretched. When the external force disappears, the stretched part will be retracted to the original shape and size. Even if some fabrics cannot be retracted immediately, they can also be ironed. Returning to the original shape, it is precisely because of this characteristic that the knit fabric clothes will show a strong sense of comfort and unrestrained freedom after being worn, thus being welcomed by the market and becoming one of the best-selling apparel fabrics. In the process of designing, professional designers will directly feel that the stretchability and resilience of knitted fabrics have a decisive influence on the design of the pattern. It is also directly related to the rationality of the layout design and the appearance of the garment. When the stretchability is strong, the designed version is easy to loosen, and when the stretchability is small, the advantages of the knitted fabric itself cannot be emphasized. In the production process of knitted garments industrialization production process, generally different amount of relaxation is determined according to different styles, and the amount of relaxation of fit-type knitted garments should be between $4 \sim 12 \mathrm{~cm}$, and the amount of relaxation of chest of tight-fitting knitted garments is generally In the following $4 \mathrm{~cm}$ is appropriate, relax the amount of chest knit garments should be more than $12 \mathrm{~cm}$ is appropriate, designers must fully understand the fabric of the specific data and physical properties, in order to carry out scientific clothing design, in order to give full play to the knitted fabric At the same time achieve the best clothing upper body effect.

\section{Methods of Knitwear Pattern Design}

Knitted garments adopt different design methods depending on the specific circumstances of garment design styles and structural types, and are mainly divided into three-dimensional construction methods and planar composition methods. When the clothing structure is relatively complicated and cumbersome, the three-dimensional composition method is used to design the pattern of the garment. When the structure of the garment is relatively simple, the planar composition method is used. The specific pattern design methods are as follows:

Specimen calculations The pattern design method, as the name implies, is mainly based on the specific requirements of the shape, size, and style of the wearer, as well as part of the detailed specification data, combined with the different size data of the measurement part and the shape of the parts, and the design of the pattern design according to the characteristics of the fabric. method. Under normal circumstances, this design method is mainly applied to the design of various types of traditional knitted garments, such as traditional style sportswear, pajamas, and the like. The pattern calculation pattern design method is simple in principle, easy to use, and has a wide operating range. It can be applied to the design of various knitted fabrics and can meet the needs of the mass production mode of factory machines. The corresponding disadvantage is that this design method can only design relatively simple traditional clothes, and cannot be used for the design of complex fashion or decorative clothing. When designing ladies short-sleeved T-shirts, special measurements shall be made on the length of clothing, the length of the bust, front and rear necks, rear necks, shoulders, shoulders, cuffs, plackets, etc. Specifications, combined with the measured data and the drawing factor of the knitted fabrics used, draw the pattern of the front and back garments and details of the pattern, and complete the complete garment design pattern.

With the increase of per capita consumption level, the aesthetics of modern society has begun to diversify. Clothing apparel not only has to satisfy basic warm and comfortable needs, but also has its own clothing to achieve the effect of personalized clothing as a whole. In the statement, the knitwear is no longer limited to home-style clothing, but it is hoped that it can be transformed into outerwear and fashionable clothing. For this reason, designers cater to consumers' willingness to consume, combined with actual life needs and modern aesthetic concepts. They want to make knitwear designs more fashionable and make their patterns more plentiful, stylish and avant-garde. In the design of knitted garments, prototypes can be used in the same way as woven garments for garment pattern design. Prototype method is relatively simple in design of the pattern design. The domestic apparel industry generally adopts the basic model of different calculation methods based on the Japanese cultural prototype or cultural prototype. The main parameters are the net bust 
parameters. Through the net bust data, scientific calculation methods are used to calculate the data of other parts of the garments. At the same time, the data of other parts can be further calculated according to the needs of different modeling, and a more comprehensive design structure can be obtained.

Take women's short-sleeved T-shirts as an example, a cultural lady's short-sleeved T-shirt style requires a certain waist design to reflect the curve of the female figure. In order to prevent the knitted garments from being stretched and deformed by a proper waist design, a design method of folding the cuffs and the hem is required. After the original version of the net bust is measured, the specifications of the length, sleeve length, shoulder width, etc. can be calculated by calculation. After the system parameter specifications are available, the pattern design can be started. The main method is to place the front and back parts of the prototype at the position of the waistline part and reserve some space between the front and back garments, and then combine the design styles corresponding to them with the specification requirements of the finished product. , Appropriately adjust the position of the garment. After the specific location is determined, the details of the collar and the sleeves are configured, and an overall paper design scheme is finally formed.

The basic pattern design method refers to the basic shape of knitted garments as a prototype, combined with the actual style of clothing and fabric characteristics required, and the appropriate adjustment of the design characteristics of its shape, and finally making a pattern of a design method. This method is based on the knitwear structure characteristics, Japan's clothing culture prototype and foreign knitted garments based on a new method of innovation, according to the different degrees of loose clothing, there are tight-fitting, fit, comfort, loose four [2]. This design method is based on the characteristics of knitting equipment for a relatively large innovation, according to the different needs of consumers wearing dress can be divided into Slim, comfortable, relaxed and other models. When designing patterns, you must master the data on the bust size, clothing length, collar width, shoulder width, sleeve length, and armhole arc length of the target layout. At the same time, the designer needs to adjust the chest width and back width according to the actual design situation, because there will be certain differences in the overall drawing. The specific pattern design application method needs to select the corresponding top coat according to different wearing comfort degrees, and then The unique characteristics of the knot style, fine-tuning the various parts of the pattern to achieve a reasonable design effect.

\section{Application and Precautions of Knitted Garment Pattern Design Method}

At the beginning of the pattern design of knitted fabrics, designers must fully understand the unique properties of the fabrics and use them as a basis for calculations and adjustments in the design, so as to ensure that the overall garment product can achieve the target design effect and the comfort degree on the clothing. , According to the data link between the characteristics and styles of fabrics, conduct a full study and calculation, and ultimately determine the most reasonable specifications for design. The edge of some knitted fabrics will be wrapped in a free state. This phenomenon is called hemming. The hemming property is influenced by the structure of the knitted fabric, the properties of the yarn (elasticity, linear density, twist and coil length) and other factors. In general, double-knitted fabrics have no curling properties, while single-side knit fabrics have more serious hemming. The style and production schedule of garments are often severely affected by the curling, resulting in low work efficiency [3]. For a fabric with greater elasticity and less resilience, the actual design needs to reduce its relaxation space appropriately to prevent loosening of the final upper body effect. Conversely, for a fabric with less elasticity. The amount of relaxation should be correspondingly increased to ensure that the knitted fabric can exhibit the excellent characteristics of comfort and restraint.

The final purpose of the pattern design of knitted garments is always to achieve the ideal garment effect. Therefore, in the process of pattern design, in addition to considering the actual elastic coefficient, fabric expansion space, design specifications, etc. The situation of shrinking or industrial shrinkage is fully taken into consideration. In addition, the amount of relaxing clothing has to consider the basic activity of the human body. The factors such as gender, age, season of 
wearing, climate, etc. of the design clothing cannot be ignored. These factors are all patterns. The design of the important factors of influence, in consideration and consideration of the specification design and adjustment in order to achieve the best design results, ensure that the design index can meet the expectations of the clothing standards. For example, when the lateral elasticity of the fabric is relatively large, the delivery space in the lateral expansion size is reduced; when the fabric has good drapability, the size specification of the length is slightly reduced, and the excessive stretching causes the version to be changed. In addition, when the pattern is converted into a wool template, the amount of corresponding seams needs to be increased according to different processes, so the corresponding sewing process design must be done before the pattern design.

In the pattern design of knitted garments, the designers must not only consider the characteristics of the fabric itself, but also take into account the impact of different production processes on the fabric, and according to the different needs of the process, the corresponding relaxation space can be reasonably added or subtracted. Make the design more reasonable overall. Different clothing production processes, different sewing routes, and different sewing machines will affect the final garment results. They will have a greater impact on the design of the pattern. In the actual operation of the garment, sometimes the fabric itself is used. Flexibility and structural design know-how to deal with excess clearance, because in general knitwear is not conceivable, it can be transferred to the side seam at the chest to float, and for the front opening of the clothing can also use the amount of chest will be part of the chest The province is transferred to the former center position. The designer needs to make different sewing values according to different equipment conditions to ensure that the results of the wool pattern and pattern design are in accordance with each other and achieve the desired design effect.

With the continuous improvement of China's national economic value, the level of people's requirements for material life is also rising further. Knitted garments are therefore on the stage and have become hot spots in the market. But because of this, we must pay more attention to this. During the process of marketization, the knitted sweaters exposed a series of problems, such as: curling deformation, dark-fabric fading and other shortcomings. In the further design process in the future, designers need to continue to specialize to solve these problems exposed, Or try to adopt more advanced technology to avoid disadvantages and ensure that the final results of garments can meet the needs of the consumer market, in line with the current society's concept of clothing and aesthetics, and thus make knitted fabrics can be more widely used in the field of garment design.

\section{Conclusion}

There are still a large number of excavation designs in knitted garments that are waiting for designers to dig out. Designers need to develop their own vision and open up ideas for design innovation and research. The method of pattern design for knitted garments is mainly based on the characteristics of different fabrics, combined with the specific production process and type requirements, to ensure that the design specifications and template design can meet the production requirements of the wool template, achieve the desired design effect, and then design. Links are refined, and innovative designs are constantly tried to ensure that the final clothing effect of knitted garments designed meets consumers' aesthetic and clothing needs. Only by satisfying consumer's design solutions can we develop in the market environment and achieve greater economic benefits. In today's diversified social development, designers should continue to think about new design methods for knitted fabrics, and strive to realize the wide application of knitted fabrics and further expand the market space.

\section{References}

[1] Jin Qianzi. Knitting apparel design exploration [D]. China Academy of Art, 2015.

[2] Peng Yu. Design and innovative application of knitwear seam [D]. Beijing Institute of Fashion, 2017. 
[3] Wu Yifeng, Zhu Qi. Knitted garment design [M]. Shanghai: Shanghai Science and Technology Press, 2011. 